\title{
Measuring the Structural and Conceptual Similarity of Folktales using Plot Graphs
}

\author{
Victoria Anugrah Lestari and Ruli Manurung \\ Faculty of Computer Science, Universitas Indonesia \\ Depok 16424, West Java, Indonesia \\ victoria.anugrah@ui.ac.id, maruli@cs.ui.ac.id
}

\begin{abstract}
This paper presents an approach to organizing folktales based on a data structure called a plot graph, which captures the narrative flow of events in a folktale. The similarity between two folktales can be computed as the structural similarity between their corresponding plot graphs. This is performed using the well-known Needleman-Wunsch algorithm. To test the efficacy of this approach, experiments are carried out using a small collection of 24 folktales grouped into 5 categories based on the Aarne-Thompson index. The best result is obtained by combining the proposed structural-based similarity measure with a more conventional bag of words vector space model, where 19 out of the 24 folktales $(79.16 \%)$ yield higher average similarity with folktales within their respective categories as opposed to across categories.
\end{abstract}

\section{Introduction}

Folktales are prevalent in almost all cultures, and are a rich and valuable part of our cultural heritage. They serve as a valuable resource for many studies into our history, sociology, and language. Analysis and classification has typically been done by folklorists, with one of the most well-known methods being the Aarne-Thompson index (Uther, 2004), which organizes folktales around the concept of motifs and themes. Folktales that are deemed to be similar based on such concepts are grouped together.

Recently, a significant amount of research has been conducted on the application of natural language processing and computational linguistics to automatically organize folktale data (Karsdorp and van den Bosch, 2013; Lobo and de Matos,
2010; Nguyen et al., 2012; Nguyen et al., 2013). Most of these approaches use the bag of words model, which measures similarity based on the number of shared features, namely words. This paper proposes a method that also takes into account the structural similarity between the sequences of events found in folktales.

The goal of our work is to develop a data structure that can be utilized to capture the structure of events, relations, and entities of a folktale. For that purpose, we propose a data structure known as a plot graph based on the work of McIntyre and Lapata (2010). The purpose of the plot graph is to record the plot movers and preserve the sequence of events in folktales. The similarity between two plot graphs can be compared by first measuring the similarity between words in corresponding vertices. The similarity between words is measured using the Wu-Palmer method of WordNetbased lexical semantic similarity (Wu and Palmer, 1994). The vertices in the plot graphs are aligned using the Needleman-Wunsch algorithm (Needleman and Wunsch, 1970).

The outline of the paper is as follows. An overview of related work is presented in Section 2, followed by discussion of the proposed plot graph data structure in Section 3 and how to measure the similarity of plot graphs in Section 4. Finally, Section 5 provides details of experimental results and Section 6 offers our conclusion and suggestions for future work.

\section{Related Work}

The Aarne-Thompson index or classification system (Uther, 2004) is a widely adopted and standardized index used by many folklorists. It was first developed by Antti Aarne in 1910, revised by Stith Thompson in 1928 and 1961, and by Hans-Jörg Uther in 2004. The system identifies a folktale through its motifs and structural patterns rather than the particular details of its characters 
actions.

In recent years, there has been a significant amount of research in trying to automatically organize folktale data using standardized indices such as the Aarne-Thompson index. In the work of Karsdorp and van den Bosch (2013), motifs in a folktale are identified using the Labeled-LDA (Latent Dirichlet Allocation), which represents motifs as distribution over words. A training model is built utilizing TF-IDF (term frequency $x$ inverse document frequency) weights as the attributes of each folktale. In the work of Lobo and de Matos (2010), a fairy tale corpus is partitioned into semantically related clusters using Latent Semantic Mapping. The model is built by first constructing a term-document matrix and then applying the singular value decomposition (SVD) matrix factorization followed by dimensionality reduction (achieved by rank reduction) to obtain a lower dimensional space that maps documents in a conceptual space. Finally, the works of (Nguyen et al., 2012; Nguyen et al., 2013) employ a supervised classification approach using a labeled corpus and various linguistic features. The former uses the SVM classifier whereas the latter employs learning-to-rank strategies.

One thing to note is that all of these existing approaches measure the similarity between folktales based on the degree of overlapping features that are typically derived from the co-occurrence of specific words or phrases. In other words, most of these approaches tend to view folktales using an approach that is known as the bag of words model. However, this approach fails to recognize the complex structure of a narrative found within a folktale, which involves plot movers and sequences of events. Since folktales are narratives, which necessarily convey a sequence of events, we argue that this narrative flow of events can possibly represent the underlying characteristic of a folktale better than bag of words methods. Furthermore, this may coincide well with the Aarne-Thompson index, as it is known that Antti Aarne focused on the morphology, or structure, of the folktale. We provide an illustrative example in Section 3.

In terms of cognitive models of similarity, we can say that the existing approaches adopt a more feature-based similarity model (Tversky, 1977), whereas we argue in favor of an approach that also takes into account structural similarity (Gentner, 1983).
In trying to formulate a model that takes into account the structural nature of narratives, one work that is of special interest is McIntyre and Lapata (2010), which develops a plot graph for the purpose of story generation. The graph captures the plot of a story by storing each entity in a vertex and each action in another vertex. Through genetic algorithm techniques, the plot graph experiences mutation and crossover with another plot graph, resulting in a new, randomized graph to generate a new story. Although the end task is significantly different, i.e. the generation of new stories, the approach to modelling plot structure is one that we adopt.

Our approach, which we will now describe in Sections 3 and 4, differs from the aforementioned works of Lobo and de Matos (2010; Nguyen et al. (2012; Nguyen et al. (2013; Karsdorp and van den Bosch (2013) in that it does not adopt a bag of words approach to capture the motif of a folktale. Rather, we build the plot graph based on the work of McIntyre and Lapata (2010), although with some modifications.

\section{Structural similarity and plot graphs}

Based on work in the area of cognitive science (Love, 2000), we propose two factors that must be considered when modelling human perception of similarity: structural similarity and conceptual similarity. Structural similarity measures the degree of isomorphism between complex objects. Conceptual similarity is a measure of relatedness between two corresponding concepts, assuming that the entities in the complex objects have been appropriately mapped.

This model can be applied to a system that has the ability to detect plot similarity the way humans do. Humans can determine whether two folktales are similar not by counting how many of the same words are shared in the folktales, but by recognizing the plot similarities of the folktales. For example, the folktales "Aladdin and the Wonderful Lamp" and "The Magic Ring" can be judged as similar. Both folktales contain magic objects (the lamp in "Aladdin and the Wonderful Lamp" and the ring in "The Magic Ring") and rag-to-riches protagonists, who meet magical helpers and marry the princess. Details such as the protagonists' names (Aladdin or Martin), the helpers' forms (a genie, a dog, or a cat), and the magic objects (a lamp or a ring) are overlooked when humans judge 
the similarities of the folktales.

\subsection{Definition of a plot graph}

We define the plot of a folktale as a sequence of events consisting of the following parts:

Action - The main word, usually a verb, that drives the course of events in a sentence. A sentence may have more than one action.

Child - A word related to an action based on its dependency relation. An action may have zero to many children.

Entity - An actor or object contained in the event. A sentence may have more than one entity.

The plot graph represents the sequence of events and the entities involved in a folktale. Thus, two plot graphs can be compared to yield a similarity score. In our experiments, we use the dependency parser (de Marneffe and Manning, 2008) and coreference resolution tool (Lee et al., 2013) found in Stanford CoreNLP library ${ }^{1}$. Our algorithm processes one sentence at a time. A sentence is parsed, and its coreferences are resolved. The actions, children, and entities are identified to be stored in the plot graph.

More formally, a plot graph is a directed acyclic graph representing a folktale that has been decomposed into actions, children, and entities. A plot graph is more predictable than a regular graph because it has a pattern, a start, and an end. Formally, a plot graph $\boldsymbol{G}$ is defined as an ordered six-tuple $\left(V_{1}, V_{2}, V_{3}, E_{1}, E_{2}, E_{3}\right)$ with the following specification:

- $V_{1}$ is a set of vertices that represent actions in the folktale. $V_{1}$ may have $n$ elements, with $n \geq 1$. An element of $V_{1}$ is called action vertex.

- $V_{2}$ is a set of vertices that represent children (words related to actions) in the folktale. $V_{2}$ may have $k$ elements, with $k \geq 1$. An element of $V_{2}$ is called child vertex.

- $V_{3}$ is a set of vertices that represent entities in the folktale. $V_{3}$ may have $m$ elements, with $m \geq 1$. An element of $V_{3}$ is called entity vertex.

- $E_{1}$ is a set of edges that links exactly two elements of $V_{1} . E_{1}$ may have $n-1$ elements. An element of $E_{1}$ is called action-action edge.

\footnotetext{
${ }^{1}$ http://nlp. stanford.edu/software/ corenlp.shtml
}

- $E_{2}$ is a set of edges that links an element of $V_{1}$ and an element of $V_{2} . E_{2}$ may have up to $n \times k$ elements. An element of $E_{2}$ is called action-child edge.

- $E_{3}$ is a set of edges that links an element of $V_{2}$ and an element of $V_{3} . E_{3}$ may have up to $m \times k$ elements. An element of $E_{3}$ is called entity-child vertex.

Figure 1 provides an illustration of the schema of a plot graph.

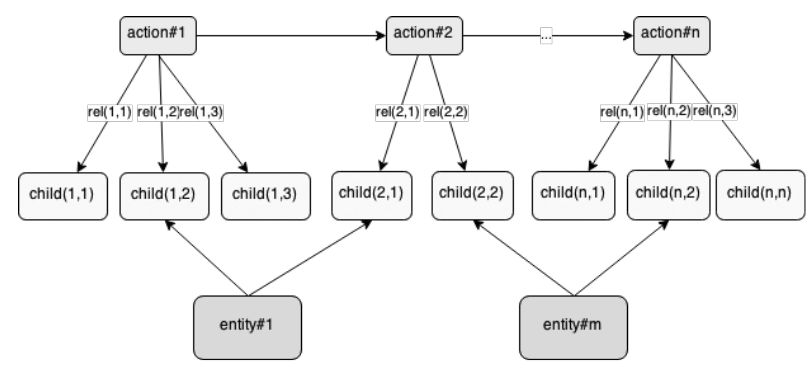

Figure 1: Schema of a plot graph

Note that there are some differences between our plot graphs as defined above with the plot graphs of McIntyre and Lapata (2010), where our plot graph is not an entity-based graph and that a vertex only contains a single event or entity.

\subsection{Construction process}

The construction of a plot graph is shown in the following example. Consider the first paragraph of the short folktale "A Friend in Need Is a Friend Indeed":

Once upon a time there lived a lion in a forest. One day after a heavy meal, it was sleeping under a tree. After a while, there came a mouse and it started to play on the lion.

With the dependency parser, we extract the dependency relations between the words in the folktale and identify the verbs, if there are any, as the actions. A dependency relation takes the form of relation(word1, word2), where word1 is the governor and word 2 is the dependent. The above paragraph yields the following relations:

advmod(lived-6, Once-1)

nsubj(lived-6, there-5)

dobj(lived-6, lion-8) 


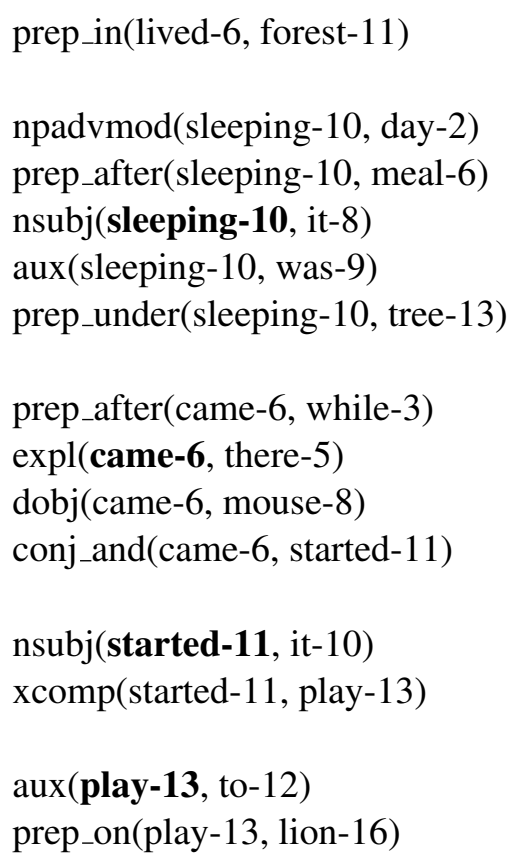

First, observing that the governors of the relations nsubj (nominal subject), expl (expletive "there"), and aux (auxiliary) are best identified as the actions of the folktale, the action words "lived", "sleeping", "came", "start", and "play" are obtained, and are used to form the action vertices.

Next, the words that are related to the actions (namely, the dependent of the relation) are obtained and identified as the children. These are used to form the child vertices, after first removing words such as "once", "there", and "was". Finally, coreference resolution is used to detect anaphoric references between the entities. Entity vertices are formed and linked to the child vertices. Entities that only occur once in the sentence are not included in the entity vertices. Thus, there are only two entities that are identified in this example, namely "the lion" in sentence 1 , and "the mouse" in sentence 3. The resulting plot graph can be seen in Figure 2.

\section{Similarity Measurement}

The similarity between two plot graphs is represented by a score in the interval $[0,1]$. A score of 0 denotes that the plot graphs are completely dissimilar, while a score of 1 denotes that the plot graphs are identical. To measure the similarity between plot graphs, first we align the action vertex sequences of the respective plot graphs.

\begin{tabular}{|c|c|c|c|c|}
\hline & & live & sleep & disturb \\
\hline & $0($ done $)$ & $-1(\longleftarrow)$ & $-2(\longleftarrow)$ & $-3(\longleftarrow)$ \\
\hline eat & $-1(\uparrow)$ & $0.29(\nwarrow)$ & $0(\nwarrow)$ & $-1(\longleftarrow)$ \\
\hline live & $-2(\uparrow)$ & $-0.71(\uparrow)$ & $0.54(\nwarrow)$ & $1(\nwarrow)$ \\
\hline rest & $-3(\uparrow)$ & $-1.71(\nwarrow)$ & $-0.38(\nwarrow)$ & $\mathbf{0 . 7 9}(\nwarrow)$ \\
\hline
\end{tabular}

Table 1: The final state of alignment matrix

Strictly speaking, computing a structural similarity mapping between two such complex expressions is an instance of the NP-hard graph isomorphism problem. However, if we observe the definition of the plot graph, we can see that it is mostly linear in structure: the sequence of event vertices forms the spine of the graph, and it is this sequence that most determines the plot. Thus, we can reduce the problem of mapping two plot graphs to a linear case of sequence matching, for which much more efficient algorithms are known.

We use the Needleman-Wunsch algorithm to obtain the maximum score of the optimal alignment. As a variation of the edit distance measurement, the Needleman-Wunsch algorithm uses a dynamic programming approach to fill two matrices: the alignment matrix and the trace-back matrix (Needleman and Wunsch, 1970).

In this work, we use the Wu-Palmer similarity measurement for the scoring matrix ( $\mathrm{Wu}$ and Palmer, 1994). If a vertex is aligned with a gap instead of another vertex, we give a gap penalty. Then we compute the word similarity between two corresponding action vertices of the respective plot graphs. We also compute the word similarity between two corresponding child vertices.

The following example shows how the Needleman-Wunsch algorithm is used to align folktales. Suppose we have two plot graphs. The first has the action vertices containing the words "live", "sleep", and "disturb". The second has the action vertices containing the words "eat", "live", and "rest". We build the scoring matrix using the Wu-Palmer similarity measurement and determine the gap penalty to be -1 . After filling the alignment matrix and the trace-back matrix according to the algorithm, we obtain the final conditions of both matrices as shown in Tables 1.

The similarity score between two plot graphs $p_{1}$ and $p_{2}$ is the sum of total action vertex similarity multiplied by a coefficient, total child vertex similarity multiplied by a coefficient, and the sum of gap penalty, as seen in Equation (1). 


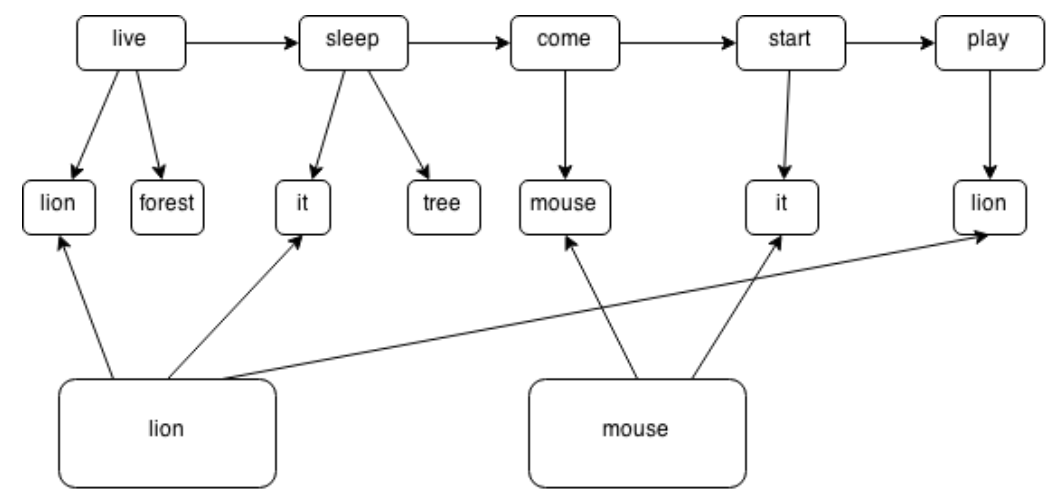

Figure 2: Plot graph representation of "A Friend in Need is a Friend Indeed"

$$
\begin{aligned}
\operatorname{sim}\left(p_{1}, p_{2}\right)= & \alpha \times \sum_{i=1}^{n} \operatorname{sim}\left(a_{1 i}, a_{2 i}\right) \\
& +\sum g+\beta \times \sum_{i=1}^{n} \operatorname{sim}\left(c_{1 i}, c_{2 i}\right)
\end{aligned}
$$

with:

$p_{1}=$ the first plot graph

$p_{2}=$ the second plot graph

$\alpha=$ coefficient for action vertex similarity

$\beta=$ coefficient for child vertex similarity

$\left(a_{1 i}, a_{2 i}\right)=$ ordered pair of action vertices from the alignment of $p_{1}$ and $p_{2}$

$g=$ gap penalty

$\left(c_{1 i}, c_{2 i}\right)=$ ordered pair of child vertices from the

alignment of $p_{1}$ and $p_{2}$

$n=$ alignment length of $p_{1}$ and $p_{2}$

To obtain a similarity score between 0 and 1 , we normalize the score from Equation (1) with a feature scaling equation, as seen in Equation (2).

$$
\begin{aligned}
& x^{\prime}=\frac{x-\min (x)}{\max (x)-\min (x)} \\
& \max (x)=\max \left(\text { length }\left(s_{1}\right), \text { length }\left(s_{2}\right)\right) \\
& \min (x)=\max (x) \times g
\end{aligned}
$$

with:

$x^{\prime}=$ transformed score

$x=$ the previous score

$s_{1}=$ the first sequence

$s_{2}=$ the second sequence

$g=$ gap penalty

Given two plot graphs, $p_{1}$ and $p_{2}$, each having length $n$, the maximum similarity score is $n$. For each alignment of action vertices, the score is 1 , leading to the maximum score, $n \times 1=n$. On the other hand, the minimum score is obtained when a plot graph with length $n$ is compared to an empty plot graph (without vertices), where each action vertex is aligned with a gap. Therefore, the minimum score is $n \times g$.

\section{Experiments}

\subsection{Parameter tuning}

The coefficients $\alpha$ and $\beta$ determine the relative importance to be placed on the similarity of the actions as opposed to the corresponding entities involved in the actions, whereas $g$ determines how much the similarity measure is willing to tolerate skips or swaps in the action sequences. To empirically determine the best values for these three values, a small experiment using 6 short folktales from an online collection of simple short stories ${ }^{2}$ is conducted. Each folktale is manually modified into 5 different paraphrases through word substitution, sentence structure changes, and insertion or deletion of words or sentences. We experiment with 3 different $\alpha$ and $\beta$ values and 3 gap penalty values for a total of 9 combinations, as can be seen in Table 2. With this scheme, we expect to see the behavior of the system when we put more weight on action vertex similarity, equal weight on both action similarity and child similarity, and more weight on child vertex similarity.

After extracting plot graphs from each folktale and their 5 paraphrases, we do a complete pairwise comparison between all plot graphs. We then average the similarity between each folktale and its 5 paraphrases, and separately we average the

\footnotetext{
${ }^{2}$ http: / / www.english-for-students.com/ Simple-Short-Stories.html
} 


\begin{tabular}{|c|c|c|c|}
\hline Experiment & $\alpha$ & $\beta$ & $g$ \\
\hline 1 & 0.7 & 0.3 & 0 \\
\hline 2 & 0.7 & 0.3 & -0.5 \\
\hline 3 & 0.7 & 0.3 & -1 \\
\hline 4 & 0.5 & 0.5 & 0 \\
\hline 5 & 0.5 & 0.5 & -0.5 \\
\hline 6 & 0.5 & 0.5 & -1 \\
\hline 7 & 0.3 & 0.7 & 0 \\
\hline 8 & 0.3 & 0.7 & -0.5 \\
\hline 9 & 0.3 & 0.7 & -1 \\
\hline
\end{tabular}

Table 2: Parameter variation for fine-tuning experiment

similarity between different folktales. We seek the parameter combination that maximizes the former value, because the paraphrases are essentially different ways of retelling the same folktale, whilst minimizing the latter value, because the similarity between genuinely different folktales should be low. In other words, we seek to find the parameter combination that maximizes the difference between these two values, as it leads to the strongest discriminatory power.

Table 3 shows the lowest similarity scores of comparison between paraphrases, the highest similarity scores of comparison between folktales, and the average scores of both comparisons.

Based on this empirical study, the values $\alpha=$ $0.3, \beta=0.7$, and $g=0$ are determined as the best parameters. It is interesting to note that the parameters that maximize perceived similarity are those that place more weight on the similarity of corresponding child nodes, i.e. the entities that are associated with the actions. We believe this may be a reflection of how humans are sensitive to higher order matches, i.e. when not only elements from one analog map to elements in the other analog, but also when their respective parents and/or children are also in correspondence (Love, 2000). In other words, analogs are perceived as similar when they have a common relational structure (Gentner, 1983).

\subsection{Comparison to bag of words}

Having concluded the parameter tuning experiment, we move on to our main experiment that seeks to observe the efficacy of plot graphs as a representation of folktales. We compare it to a representative bag of words method, and also a combination where the average between both similarities is used. For this experiment, we use 24 folktales from the Fairy Books of Andrew Lang, available under Project Gutenberg ${ }^{3}$. We classify the folktales into 5 groups according to the AarneThompson index. The groups are as follows:

- Supernatural Adversaries - Bluebeard; Hansel and Gretel; Jack and the Beanstalk; Rapunzel; The Twelve Dancing Princesses.

\section{- Supernatural or Enchanted Relatives -} Beauty and the Beast; Brother and Sister; East of the Sun, West of the Moon; Snow White and Rose Red; The Bushy Bride; The Six Swans; The Sleeping Beauty.

- Supernatural Helpers - Cinderella; Donkey Skin; Puss in Boots; Rumpelstiltskin; The Goose Girl; The Story of Sigurd.

- Magic Objects - Aladdin and the Wonderful Lamp; Fortunatus and His Purse; The Golden Goose; The Magic Ring.

- Other Stories of the Supernatural - Little Thumb; The Princess and the Pea.

Note that it does not matter if the groups are unequal in size, since we are only analyzing the similarity scores of folktales between groups and within groups.

As before, the 24 folktales are automatically converted into plot graphs, and a pairwise comparison between all the plot graphs is conducted using the similarity measure described in Section 4 . The longest of these folktales, Beauty and the Beast, yields 1,208 action vertices, 2,229 child vertices, and 694 entity vertices, whereas the shortest folktale, Jack and the Beanstalk, yields 633 action vertices, 1,260 child vertices, and 497 entity vertices.

For the bag of words comparison, the folktales are first converted into vectors of words. Each component of the vector is the term frequency (TF) of the word in the folktale. We compare the vectors with each other using the cosine similarity measure:

\footnotetext{
${ }^{3}$ http://www . gutenberg . org/ebooks/30580
} 


\begin{tabular}{|c|c|c|c|c|c|c|c|c|c|c|}
\hline & \multicolumn{3}{|c|}{$\alpha=0.7, \beta=0.3$} & \multicolumn{3}{|c|}{$\alpha=0.5, \beta=0.5$} & \multicolumn{3}{|c|}{$\alpha=\mathbf{0 . 3}, \beta=0.7$} \\
\hline & & $g=-1$ & $g=-0.5$ & $g=0$ & $g=-1$ & $g=-0.5$ & $g=0$ & $g=-1$ & $g=-0.5$ & $g=0$ \\
\hline Comparison & Avg. & 0.83 & 0.80 & 0.74 & 0.83 & 0.80 & 0.73 & 0.83 & 0.79 & 0.71 \\
\hline between paraphrases & Min. & 0.69 & 0.61 & 0.53 & 0.69 & 0.60 & 0.49 & 0.68 & 0.58 & 0.45 \\
\hline Comparison & Avg. & 0.37 & 0.30 & 0.15 & 0.41 & 0.32 & 0.12 & 0.45 & 0.33 & 0.09 \\
\hline between folktales & Max. & 0.55 & 0.45 & 0.25 & 0.55 & 0.43 & 0.20 & 0.55 & 0.42 & 0.16 \\
\hline \multicolumn{2}{|l|}{ Min. - Max. } & 0.14 & 0.16 & 0.28 & 0.14 & 0.17 & 0.29 & 0.13 & 0.16 & 0.29 \\
\hline \multicolumn{2}{|l|}{ Diff. between averages } & 0.46 & 0.50 & 0.59 & 0.42 & 0.48 & 0.61 & 0.38 & 0.46 & 0.62 \\
\hline
\end{tabular}

Table 3: Results of parameter tuning

$$
\cos (\angle \vec{A} \vec{B})=\frac{\vec{A} \cdot \vec{B}}{\|\vec{A}\|\|\vec{B}\|}
$$

with $\vec{A}$ and $\vec{B}$ denoting vectors of words.

The experiment is done under the expectation that the average similarity scores between folktales within a group are higher than the average similarity scores between folktales across the different groups. In other words, "Aladdin and the Wonderful Lamp" should have a higher similarity score to "The Magic Ring", since both of them belong to the Magic Objects group, than to "Beauty and the Beast", which belongs to the Supernatural or Enchanted Relatives.

When using the plot graph-based similarity scores, out of the 24 folktales, only 10 folktales $(41.67 \%)$ yield higher average scores within their respective groups than when compared to folktales across groups. When using the bag of words comparison method, 14 folktales (58.33\%) yield higher scores in the "within group" comparison. However, the combination experiment yields the best result, with 19 folktales $(79.16 \%)$ yielding higher scores in the "within group" comparison. The details of the experiment result can be seen in Table 4. The shaded cells show instances of folktales that yield a higher score for the "within group" comparison as opposed to the "across group" comparison. Note that no such instances are found in the last group, which can probably be explained due to it being the catch-all "Other stories" group.

The proposed plot graph similarity does not perform as well as the bag of words approach. Upon further inspection of the data, we note that most instances that do not fit our expectation occurs due to limitations in the automatic plot graph construction method that we have implemented rather than the theoretical model itself. We note several problems. The way the plot graph is constructed based on the Stanford dependency parser can cause the system to judge two semantically identical sentences with different syntactic structures as different. For example, the sentence "If I am hungry, I will eat" will generate a plot graph consisting of action vertices hungry followed by eat, whereas the sentence "I will eat if I am hungry" will generate a plot graph eat followed by hungry ${ }^{4}$. Similarly, two semantically similar but structurally different sentences can generate significantly different plot graphs and, therefore, produce a lower similarity score. For example, the sentence "The lion is very hungry" will generate a plot graph with an action vertex hungry and a child vertex lion, whereas the sentence "The lion has an extreme hunger" will generate a plot graph with an action vertex have and child vertices lion and hunger. When the plot graphs are compared, the action vertices hungry and have will be compared, yielding a low similarity score.

Nevertheless, the best scenario is obtained when the similarity score of two folktales is the average of their plot graph similarity and bag of words cosine similarity. This suggests that the information in the two representations is complementary, and that both structural and feature-based similarity models may play a role in organizing folktales.

\section{Summary}

In this paper we have proposed a data structure called a plot graph to represent folktales. This plot graph maintains the sequence of events in the folktale by preserving the words and their relations to each other. The aim is to facilitate a similarity measure that takes into account both structural and conceptual similarity, as humans are sensitive to higher order relational matching.

From our experiments, the best scenario is obtained when the similarity score of two folktales is the average of their plot graph similarity and bag of words cosine similarity. This suggests that the

\footnotetext{
${ }^{4}$ The Stanford dependency parser identifies the word "hungry" as the governor of the dependent word "I".
} 


\begin{tabular}{|c|c|c|c|c|c|c|c|}
\hline \multirow{2}{*}{ Group } & \multirow{2}{*}{ Title } & \multicolumn{2}{|c|}{ Plot Graph } & \multicolumn{2}{|c|}{ Bag of words } & \multicolumn{2}{|c|}{ Combination } \\
\hline & & Within & Across & Within & Across & Within & Across \\
\hline \multirow{5}{*}{$\begin{array}{l}\text { Supernatural } \\
\text { Adversaries }\end{array}$} & Bluebeard & 0.1000 & 0.1037 & 0.8629 & 0.8618 & 0.4814 & 0.4586 \\
\hline & Hansel and Gretel & 0.1075 & 0.1157 & 0.8492 & 0.8630 & 0.4783 & 0.4894 \\
\hline & Jack and the Beanstalk & 0.1050 & 0.1110 & 0.9050 & 0.8891 & 0.5050 & 0.5001 \\
\hline & Rapunzel & 0.1000 & 0.1047 & 0.8790 & 0.8575 & 0.4895 & 0.4571 \\
\hline & The Twelve Dancing Princesses & 0.1125 & 0.1073 & 0.8808 & 0.8631 & 0.4966 & 0.4610 \\
\hline \multirow{7}{*}{$\begin{array}{l}\text { Supernatural } \\
\text { or Enchanted } \\
\text { Relatives }\end{array}$} & Beauty and the Beast & 0.0767 & 0.0705 & 0.8803 & 0.8605 & 0.4785 & 0.4397 \\
\hline & Brother and Sister & 0.1233 & 0.1135 & 0.8881 & 0.8722 & 0.5057 & 0.4654 \\
\hline & East of the Sun, West of the Moon & 0.1117 & 0.1012 & 0.8914 & 0.8571 & 0.5015 & 0.4525 \\
\hline & Snow White and Rose Red & 0.1200 & 0.1165 & 0.8650 & 0.8566 & 0.4925 & 0.4865 \\
\hline & The Bushy Bride & 0.1200 & 0.1182 & 0.8862 & 0.8739 & 0.5031 & 0.4960 \\
\hline & The Six Swans & 0.0925 & 0.1100 & 0.9006 & 0.8662 & 0.5020 & 0.4881 \\
\hline & The Sleeping Beauty & 0.1125 & 0.1194 & 0.8990 & 0.8918 & 0.5087 & 0.5056 \\
\hline \multirow{6}{*}{$\begin{array}{l}\text { Supernatural } \\
\text { Helpers }\end{array}$} & Cinderella & 0.1180 & 0.1144 & 0.8150 & 0.8306 & 0.4665 & 0.4725 \\
\hline & Donkey Skin & 0.1040 & 0.1122 & 0.8873 & 0.9025 & 0.4956 & 0.5074 \\
\hline & Puss in Boots & 0.1175 & 0.1095 & 0.8170 & 0.8486 & 0.4672 & 0.4551 \\
\hline & Rumpelstiltskin & 0.0750 & 0.0858 & 0.8467 & 0.8569 & 0.4609 & 0.4478 \\
\hline & The Goose Girl & 0.1240 & 0.1178 & 0.8617 & 0.8624 & 0.4928 & 0.4643 \\
\hline & The Story of Sigurd & 0.1080 & 0.1178 & 0.8516 & 0.8670 & 0.4800 & 0.4664 \\
\hline \multirow{4}{*}{$\begin{array}{l}\text { Magic } \\
\text { Objects }\end{array}$} & Aladdin and the Wonderful Lamp & 0.0975 & 0.091 & 0.8958 & 0.8664 & 0.4946 & 0.4559 \\
\hline & Fortunatus and His Purse & 0.1133 & 0.1185 & 0.8945 & 0.8306 & 0.5039 & 0.4519 \\
\hline & The Golden Goose & 0.1033 & 0.1155 & 0.9006 & 0.8529 & 0.50123 & 0.4611 \\
\hline & The Magic Ring & 0.1033 & 0.1040 & 0.9120 & 0.8960 & 0.5077 & 0.4762 \\
\hline \multirow{2}{*}{ Other Stories } & Little Thumb & 0.0300 & 0.1214 & 0.7444 & 0.8562 & 0.3872 & 0.4675 \\
\hline & The Princess and the Pea & 0.0300 & 0.0405 & 0.7444 & 0.7844 & 0.3872 & 0.3945 \\
\hline
\end{tabular}

Table 4: Experimental Results

information in the two representations is complementary, and that both structural and feature-based similarity models play a role in organizing folktales.

An analysis of the experimental results reveal that the parsed dependency relations are still too sensitive towards syntactic variations, thus more work must be carried out to reliably produce plot graphs that are able to abstract away from these variations and to represent the structural properties of the narrative in a more consistent fashion. For future work, one approach that can be explored is the use of frame vertices using Semantic Role Labeling techniques (Gildea and Jurafsky, 2002), as this can further abstract away from syntactic variations.

Finally, it would also be interesting to see how to incorporate manually richly-annotated narratives, e.g. (Elson and McKeown, 2010).

\section{References}

Marie-Catherine de Marneffe and Christopher D. Manning. 2008. The stanford typed dependencies representation. In Proceedings of the workshop on CrossFramework and Cross-Domain Parser Evaluation, pages 1-8, Manchester, UK.

David K. Elson and Kathleen R. McKeown. 2010. Building a bank of semantically encoded narratives. In Nicoletta Calzolari (Conference Chair), Khalid
Choukri, Bente Maegaard, Joseph Mariani, Jan Odijk, Stelios Piperidis, Mike Rosner, and Daniel Tapias, editors, Proceedings of the Seventh International Conference on Language Resources and Evaluation (LREC'10), Valletta, Malta. European Language Resources Association (ELRA).

Dedre Gentner. 1983. Structure-mapping: A theoretical framework for analogy. Cognitive Science, $7(2): 155-170$.

Daniel Gildea and Daniel Jurafsky. 2002. Automatic labeling of semantic roles. Computational Linguistics, 28(3):245-288.

Folgert Karsdorp and Antal van den Bosch. 2013. Identifying motifs in folktales using topic models. In Proceedings of the 22nd Annual Belgian-Dutch Conference on Machine Learning, pages 41-49, Nijmegen, Netherlands.

Heeyoung Lee, Angel Chang, Yves Peirsman, Nathanael Chambers, Mihai Surdeanu, and Dan Jurafsky. 2013. Deterministic coreference resolution based on entity-centric, precision-ranked rules. Computational Linguistics, 39(4):885-916.

Paula Cristina Vaz Lobo and David Martins de Matos. 2010. Fairy tale corpus organization using latent semantic mapping and an item-to-item top-n recommendation algorithm. In Proceedings of the 2010 Language Resources and Evaluation Conference (LREC 2010), Valletta, Malta.

Bradley C. Love. 2000. A computational level theory of similarity. In Proceedings of the 22nd Annual Meeting of the Cognitive Science Society, pages 316-321, Philadelphia, USA. 
Neil McIntyre and Mirella Lapata. 2010. Plot induction and evolutionary search for story generation. In Proceedings of the 48th Annual Meeting of the Association for Computational Linguistics, pages 1562 1572, Uppsala, Sweden. Association for Computational Linguistics.

Saul B. Needleman and Christian D. Wunsch. 1970. A general method applicable to the search for similarities in the amino acid sequence of two proteins. Journal of Molecular Biology, 48:443-453.

Dong Nguyen, Dolf Trieschnigg, Theo Meder, and Mariët Theune. 2012. Automatic classification of folk narrative genres. In Proceedings of KONVENS 2012 (LThist 2012 workshop), pages 378-382, Vienna, Austria.

Dong Nguyen, Dolf Trieschnigg, and Mariët Theune. 2013. Folktale classification using learning to rank. In Proceedings of the 35th European Conference on IR Research (ECIR 2013), pages 195-206, Moscow, Russia.

Amos Tversky. 1977. Features of similarity. Psychological Review, 84(4):327-352.

Hans-Jörg Uther. 2004. The Types of International Folktales: A Classification and Bibliography. Academia Scientiarum Fennica, Helsinki, Finland.

Zhibiao Wu and Martha Palmer. 1994. Verb semantics and lexical selection. In Proceedings of the 32nd Annual Meeting on Association for Computational Linguistics, pages 133-138, Stroudsburg, USA. 